(C) 2018 IEEE. Personal use of this material is permitted. Permission from IEEE must be obtained for all other uses, in any current or future media, including

reprinting/republishing this material for advertising or promotional purposes, creating new collective works, for resale or redistribution to servers or lists, or reuse of any copyrighted component of this work in other work 


\title{
Experimental Study of Microwave Streamer Discharge Ignition of Premixed Air/Fuel Mixtures
}

\author{
Mikhail Bulat, Pavel Bulat, Petr Denissenko, Igor Esakov, Lev Grachev, Konstantin Volkov, Igor Volobuev
}

\begin{abstract}
A subcritical microwave streamer discharge is used to initiate ignition of premixed air/fuel mixture. The streamer is arising on the internal surface of the dielectric tube using a passive vibrator in a single pulse regime at atmospheric pressure and temperature. The propagation speed of the combustion front in the quartz cylindrical tube filled by the air/propane mixture is analyzed experimentally. Ignition of lean fuel mixtures by a streamer has been demonstrated at atmospheric pressure. The performed studies showed that the streamer discharge, which creates a multitude of ignition points, provides practically instantaneous ignition of the mixture in the entire volume, where the streamers reach. Measurements have shown that the front velocity increases with increasing discharge length. The speed of streamer-induced combustion has been shown to be higher compared to that initiated by a spark. The combustion efficiency has also been shown to be higher when using the microwave streamer ignition.
\end{abstract}

Index Terms-streamer discharge, plasma applications, microwave technology, ignition, combustion, air/fuel mixture.

\section{INTRODUCTION}

$\mathbf{R}$ EDUCTION of the combustion chamber volume, improvement of fuel and air mixing, increase of flame speed are important for design and optimization of gas turbine engines, internal combustion engines and other propulsion systems. The ignition system has always posed problems in commercial applications [1]. Conventional spark plug systems have several limitations including inability to ignite lean or diluted mixtures (as mixtures become leaner, they grow more difficult to ignite), localized ignition, cathode erosion. Many experimental, theoretical and numerical studies have been performed for the past years, and various ignition systems (e.g., electric discharge, microwave discharge, laser radiation) have been tested to achieve simultaneous space ignition via multiple ignition points throughout the combustion chamber to establish a high-performance ignition method [2], [3], [4], [5], [6], [7].

The combustible mixture is ignited with the addition of a local ignition energy sources such as heat, chemically active molecules or radicals and an electric spark into the chamber of premixed mixture provided that the minimum ignition

M. Bulat and P. Bulat are with Baltic State Technical University, 190005, St Petersburg, Russia.

P. Denissenko is with University of Warwick, CV4 7AL, Coventry, United Kingdom.

I. Esakov and L. Grachev are with Moscow Radiotechnical Institute of Russian Academy of Sciences, 117519, Moscow, Russia.

K. Volkov is with Kingston University, SW15 3DW, London, United Kingdom.

I. Volobuev is with ITMO University, 197101, St Petersburg, Russia.

Manuscript received March 22, 2018; revised June 6, 2018. energy requirement is satisfied. The non-conventional ignition technique such as laser-induced ignition is widely used due to its advantages over conventional ignition systems for the reliable and stable operation of combustion devices [8]. The initiation of flame in many points through the mixture can be done almost simultaneously by using laser-induced ignition technique in the multiple locations inside the combustion chamber [9]. It is found that the total burning time is reduced significantly with the adoption of multi-point ignition technique which enhances the combustion efficiency as well [10]. The successful ignition of a combustible mixture not only initiates the combustion event but also influences the subsequent combustion process.

Plasma-assisted combustion is a promising technique to improve engine efficiency, reduce emissions and enhance fuel reforming [1]. Ignition and combustion control using cold and non-thermal plasmas appearing in microwave discharges has become a major topic of interest [11], [12]. Advances in microwave sources, experimental diagnostic techniques, numerical simulation and computing power have allowed significant progress in the understanding of plasma-assisted ignition and combustion.

The idea of using plasma methods of fuel ignition is based on non-equilibrium generation of chemically active particles, accelerating the combustion process. The methods of generation of chemically active discharge plasma and mechanisms of ignition and combustion controlled by non-equilibrium plasma are provided in [12]. Mechanisms for the acceleration of combustion under the action of a non-equilibrium plasma are actively discussed including the generation of atomic oxygen and other chemical radicals [13], development of molecules of singlet delta-oxygen [14], chain ion-molecular reactions with intermediate radicals [15]. The possibility of a nonequilibrium electric discharge is experimentally demonstrated in [16], [17], [18], [19]. The results of experimental and numerical analysis of plasma-assisted combustion at a normal and high pressures and temperatures are presented in [20]. The spatial and temporal dynamics of a pulsed microwave plasma is studied in [21]. The effects of the pulse repetition frequency on the plasma ignition dynamics are discussed. The experimental results highlight the effects of the pulse repetition frequency on the discharge dynamics. Electrical breakdown resulting in the ignition of a low-pressure low-current glow discharge in long (length much larger than the diameter) tubes is studied in [22].

Among the various types of discharges studied, the microwave streamer discharge has recently demonstrated promising characteristics for ignition at low initial temperatures [23]. 
The streamer discharge looks as a chaotic structure of plasma channels (filaments). Their characteristic diameter is about a fraction of millimeter, and a characteristic distance between the channels is a fraction of wavelength. A streamer filament divides itself into several branches that connect to each other, forming a net of thin plasma filaments, whose characteristic length is related to electrodynamic resonance effects. A local initiation of such a discharge is provided by special facilities. The streamer discharge spreads in a considerable volume, and power is much smaller than the critical one (subcritical microwave discharge). The diameter of the plasma channels is a key parameter that determines ignition. If it is too large, ignition becomes more difficult or even impossible, whatever the energy released. It is more efficient for ignition to increase the power by varying the thickness of the plasma channels than by releasing more energy.

Lean mixtures can be completely burnt using streamer discharge with a reasonable released energy, while spark remains inefficient at very low equivalence ratios. Nanosecond microwave discharge is able to induce several points of ignition, while spark ignition always remains localized at a single point. Ignition time is also reduced with nonequilibrium discharge like streamer discharge. A thermal spark promotes neutral heating by maintaining the electrical current over a long period, but it is less efficient in the end [24].

In this study, possibilities of the use of microwave radiation to initiate combustion of premixed air/fuel mixtures in the quartz cylindrical tube are investigated experimentally. The streamer discharge is formed by a field with the electrical field strength which is smaller than the minimum pulse intensity leading to the air breakdown. Electromagnetic beam has sizes about tens of wavelength of microwave radiation along its propagation and about some units of a wavelength in the radial direction. The discharge is ignited in a space far away elements forming electromagnetic beam.

\section{Diagnostic Method}

Experimental setup and technique have been developed at the Moscow Radiotechnical Institute (MRTI) of Russian Academy of Sciences (RAS). These were adopted for the current study dealing with microwave-induced combustion in the quartz cylindrical tube.

Some details of the experimental setup related to initiation of a microwave discharge are shown in the Figure 1. The test rig includes a microwave generator that produces electromagnetic waves with a frequency $3.4 \mathrm{GHz}$ corresponding to the wavelength $8.9 \mathrm{~cm}$ (Figure 1a). Duration of the microwave pulse is $40 \mu \mathrm{s}$, and the power of the beam varies from $10^{2}$ to $10^{6} \mathrm{~W}$. Electromagnetic wave passes polystyrene lens and falls onto the axisymmetric metal concave spherical mirror with $450 \mathrm{~mm}$ curvature radius, $685 \mathrm{~mm}$ diameter and 158 $\mathrm{mm}$ depth (Figure 1b). Electromagnetic wave reflected from the mirror is focused on the centreline on $225 \mathrm{~mm}$ distance from the center of spherical mirror surface or $67 \mathrm{~mm}$ distance from the mirror mouth. So, quasi-optical beam with relatively sharp boundaries is formed.

The quartz tube is placed in the focus region of the quasioptical beam. One end of the tube is closed, and another

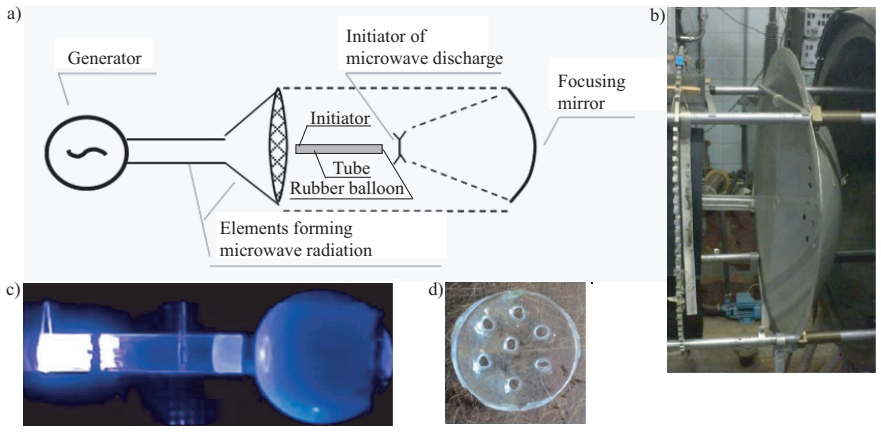

Fig. 1. Details of the experimental test rig.

one is open. The tube length is $500 \mathrm{~mm}$, and its internal diameter is $30 \mathrm{~mm}$. A rubber balloon is attached to the open end of the tube (Figure 1c) separating air/fuel mixture from the surrounding. A polystyrene protective membrane is attached to the open end of the tube (Figure 1d) preventing balloon damage at air pumpage from the tube. A small tube is connected to the membrane which is used to pumped out the air and to supply air/propane mixture. Fuel and oxidizer concentrations are controlled by varying partial pressures. The measurements are performed with stoichiometric, lean (fuelto-air ratio is 0.87 ) and rich (fuel-to-air ratio is 1.33) mixtures. The discharge initiator is placed on $27 \mathrm{~mm}$ distance from the closed end of the tube.

Development of the streamer and the propagation of the flame front has been recorded using a monochrome high-speed camera Phantom v.2511 with the frame rate of up to 750,000 frames/s and the color Nikon1 J2 camera at a frame rate of 1200 frames/s. The speed of the flame front propagation and the induction time from the moment of the discharge to the beginning of combustion reaction have been measured.

To study the effect of microwave field intensity on the type of generated discharge and the nature of combustion, the initiator is placed in the focus of the mirror and then shifted along the axis. The results of measurements of the electric field strength are shown in the Figure 2a. In the tests, power and energy of microwave beam remain the same. To vary energy of the microwave discharge, the discharge initiator shifts relative to the focus, and the electric field strength and energy release are found from the graph. The length of the streamer discharge strongly depends on the strength of electric field (Figure 2b).

The $x$ axis is parallel to the magnetic component, and $z$ axis is parallel to the electric component of electromagnetic field. The $y$ axis is parallel to the direction of the beam propagation. Near the focus the electric field distribution is approximated by the Gaussian law with characteristic sizes $x_{0}=2.5 \mathrm{~cm}$ and $z_{0}=5.2 \mathrm{~cm}$. Maximal electric field in the focus equals to $E_{0}=7 \times 10^{3} \mathrm{~V} / \mathrm{cm}$.

The electrical field strength is related to the power of the beam as $P=E_{0}^{2} S /\left(2 Z_{0}\right)$, where $Z_{0}=\mu_{0} c=120 \pi$ is the characteristic resistance of a free space [Ohm], $\mu_{0}$ is the magnetic constant, $c$ is the speed of light. The cross section of the beam is ellipse, and the characteristic area of the beam is $S=0.5 \pi y_{0} z_{0}$. The power of the beam is $P=620 \mathrm{kV}$ assuming that $E_{0}=4.8 \mathrm{kV} / \mathrm{cm}$. The duration of the pulse 

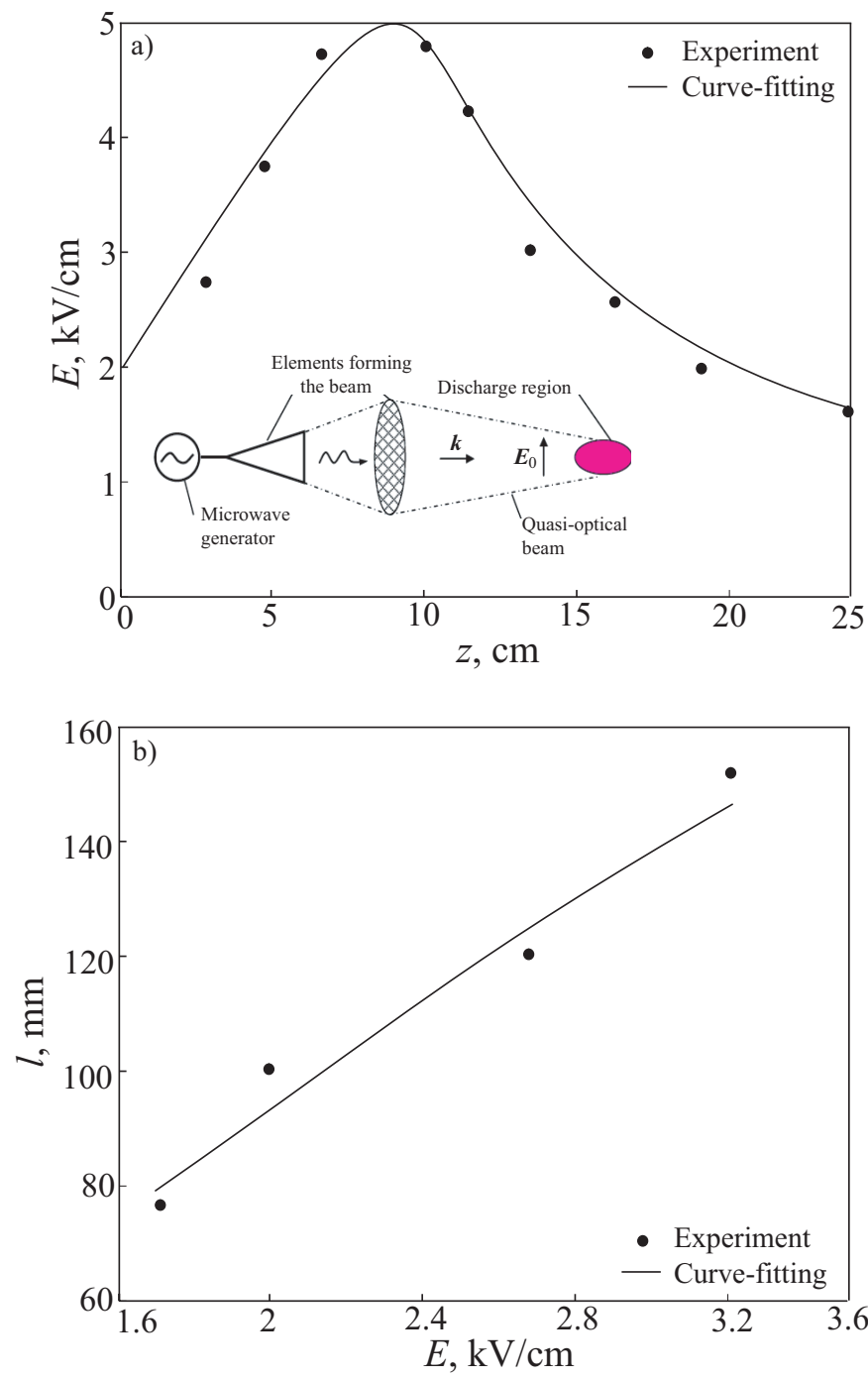

Fig. 2. Electric field strength as a function of distance from the mirror edge (a) and length of the streamer discharge as a function of electric field strength (b).
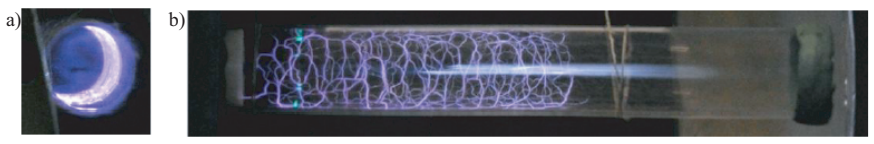

Fig. 3. Streamer discharge initiated on the internal surface of the quartz tube: face view (a) and side view (b).

is $40 \mu \mathrm{s}$, and energy of the beam is $Q=24 \mathrm{~J}$. The power and energy remain constant in the experiments, and discharge initiator is shifted with respect to the focus. Then, electrical field strength is found from the Figure 2, and energy of the beam is calculated.

The streamer discharge is induced on the ends of the initiator representing the metallic net with squared cells and covering internal cylindrical surface of the quartz tube (Figure $3 a$ ) and propagates towards the beam (Figure $3 b$ ). Blue points on the ends of initiator are visible in the Figure $3 \mathrm{a}$, and the streamer discharge (purple color) propagates from these points.

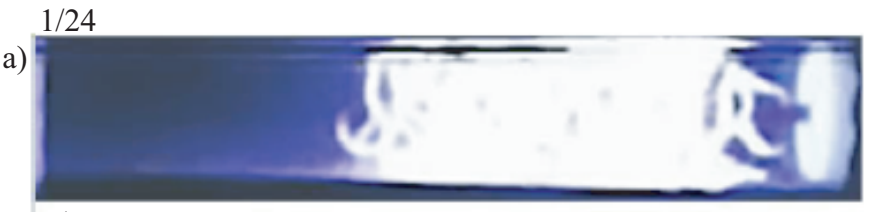

$2 / 24$

b)

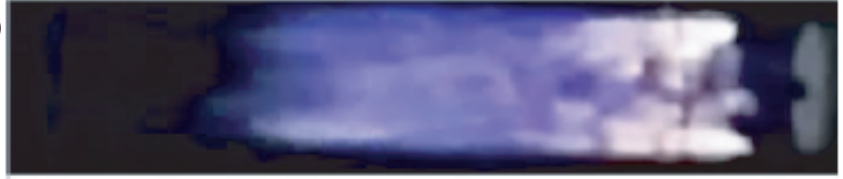

$3 / 24$

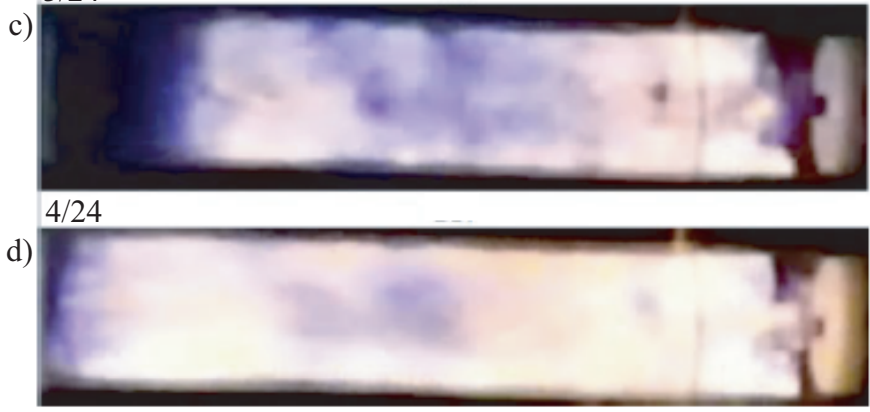

Fig. 4. Development of combustion front for $E_{0}=2.4 \mathrm{kV} / \mathrm{cm}$ and length of streamer discharge $110 \mathrm{~mm}$. The camera speed is 24 frames/s.

\section{RESUlTS AND Discussion}

The subcritical streamer discharge is formed by electric field with the intensity smaller than the minimum pulse intensity leading to the air breakdown. Streamer discharge ignition of air/propane mixture with excess oxidant ratio greater than flammability limit under normal conditions is studied. Analysis of the video tapes reveals the great variety of shapes in the discharge phenomena observed in the experiments.

Combustion starts along entire length of each filament. Volumetric ignition takes place not in the whole volume, in which streamer propagates. Ignition zone occupies about one third of the streamer discharge from the end of the tube (Figure 4). Discharge glow is the brightest in this region. At the strength of electric field $E_{0}=2.0 \mathrm{kV} / \mathrm{cm}$ the flame front has a propagation speed of $15 \mathrm{~m} / \mathrm{s}$ in the region occupied by of streamer discharge. Then, the flame shape becomes flatter, and its speed is lowered to about $2.5-5 \mathrm{~m} / \mathrm{s}$. The speed of combustion front lowers in 3 times if the strength of electric field drops to $1.8 \mathrm{kV} / \mathrm{cm}$. The process is accompanied by a bright flash and generation of sound.

The length of the streamer discharge is controlled by shifting the tube from the microwave beam focus. The length of discharge is about $180 \mathrm{~mm}$ if the initiator is located at the focus. It decreases shifting the initiator. Measurements of the length of discharge and propagation speed of the combustion front is based on the post-processing of the videos taken with 10000 frames/s (Figure 5). The first frame shows the glow from the streamer discharge. Dark vertical lines correspond to the external elements of the experimental setup.

High-speed camera with a frame speed of 1200 frames/s allows to measure the flame propagation speed with the ignition of the mixture induced by deep subcritical streamer 

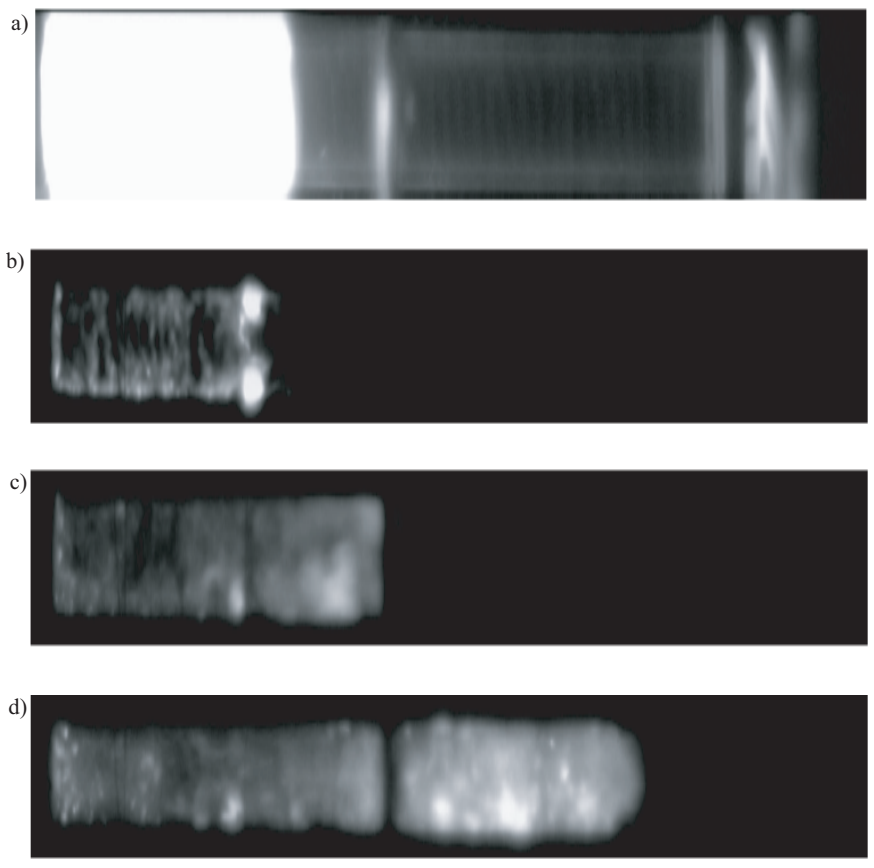

Fig. 5. Development of combustion front. The camera speed is 8900 frames/s.

discharge. Spatial-temporal diagrams, typical one is presented in the Figure 6, are used in post-processing of the measured data. There are two slopes in the ignition kernel growth rate. The steeper slope persists while the discharge pulse burst is active, and represents the growth of the plasma volume as it is transported downstream by the flow. The second slope represents the ignition kernel expansion in the absence of the plasma. This is only a function of the flame speed, and is not affected by the plasma volume. In this case, the length of tube occupied by the streamer discharge is about $15 \mathrm{~cm}$, and flame propagation speed is about $160 \mathrm{~m} / \mathrm{s}$. The flame front propagates with high speed while combustion waves from the discharge initiators merge on the tube centreline. Then, propagation speed decreases due to appearance of the rarefaction wave propagating towards closed end. A flame propagating from the open channel end to the closed one interacts with acoustic waves reflected form the closed end, leading to folding of the flame shape.

The velocity of the flame front has been measured with an array of photodiodes set along the tube wall and, independently, from photographic records. The experimental results on flame propagation speed are presented in the Figure. 7. Circles correspond to stoichiometric mixture, bullets correspond to rich mixture (fuel-to-air ratio is 1.33) and squares correspond to lean mixture (fuel-to-air ratio is 0.87 ). Vertical lines show experimental uncertainties. Main results were obtained for stoichiometric mixture. Some results for lean and rich mixtures are included for demonstrating purposes. The probability of ignition is found by attempting to ignite the mixture six times for each condition and recording how many ignition events were successful. The measurement uncertainties are calculated from the standard deviation of the test images. Dependance of the flame propagation speed on the length of streamer

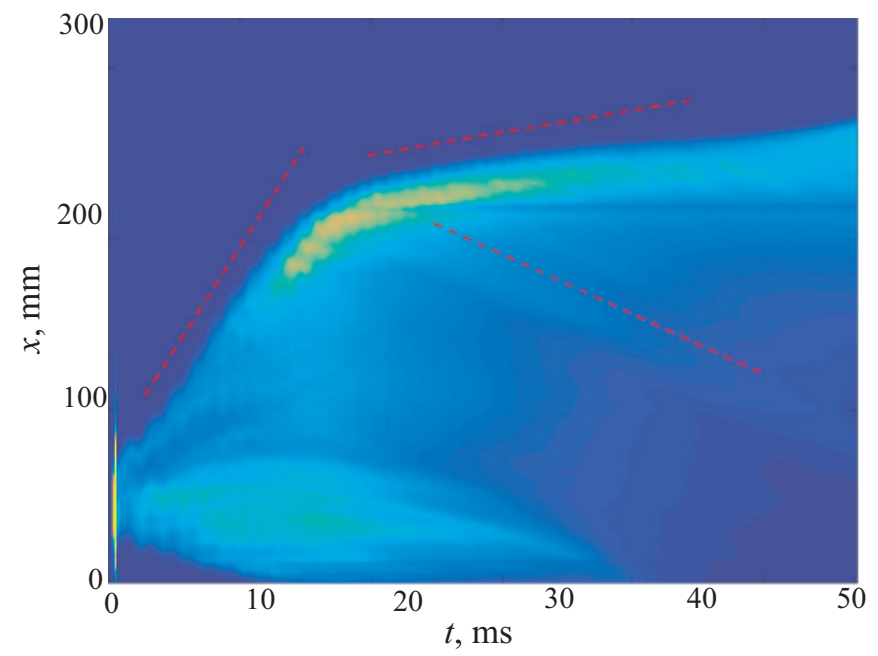

Fig. 6. Typical spatial-temporal diagram used for measurement of propagation speed of the flame (dependance of coordinate of front location on time).

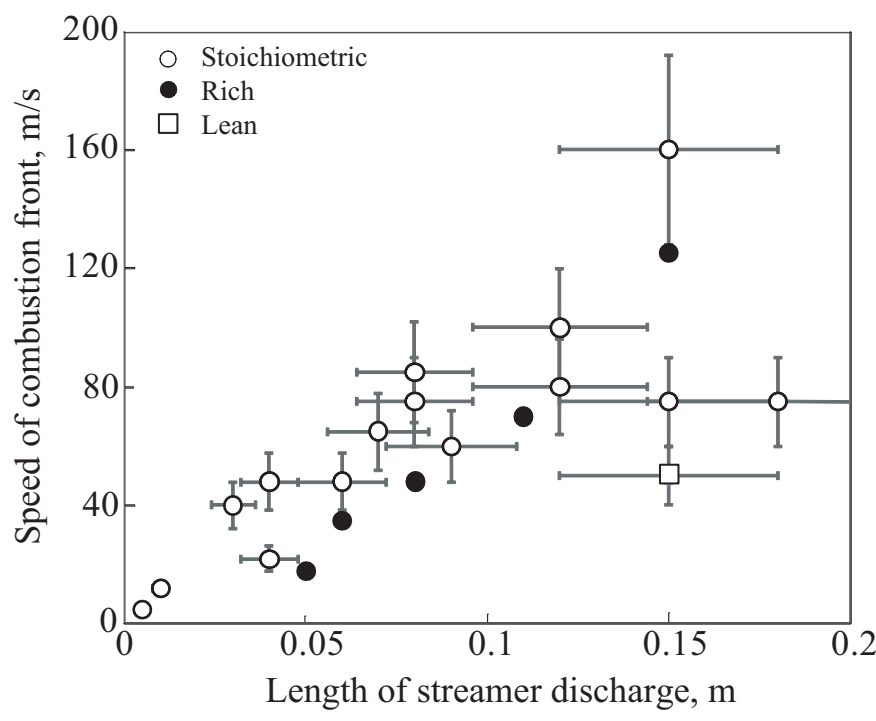

Fig. 7. Dependance of the propagation speed of flame front in the quartz tube, filled by air/propane mixture, on the length of the streamer discharge, initiated on the internal surface of the tube.

discharge is close to the linear.

The main advantage of a plasma igniter in comparison to conventional spark plug is in much higher plasma plume volume and velocity. That allows deeper penetration of the high reactive plasma plume into the combustion zone for more reliable ignition. The higher propagation speed is the result of the higher flame surface area evolved from the larger ignition areas. The flame propagation speed becomes very high due to the enlargement of flame surface area caused by the larger ignition area, and consequently, flame propagation time in the channel is reduced.

The ignition by a streamer discharge changes the nature of combustion. The rate of combustion and combustion efficiency increase by times, and no soot is formed. The indirect evidence suggests that the ignition by the microwave discharge is of the non-thermal nature. The advantages of igniting the fuel 
mixture by streamer discharge is attributed to the ultraviolet radiation emitted by oxygen atoms subjected to the discharge. The ultraviolet radiation generation causes formation of the non-equilibrium cold plasma with avalanche increase in the number of free electrons.

\section{CONCLUSION}

Ignitable area becomes one of the key parameters in addition to minimum ignition energy. The streamer discharge produces multiple filaments that can initiate ignition along the plasma channels, providing a volumetric ignition process as the streamers sweep a much larger volume compared to the conventional spark ignition. The effect of ignition surface area on the propagation of a premixed flame is investigated experimentally.

A stable ignition of the premixed air/propane mixture with the deep subcritical streamer discharge initiated on the internal surface of quartz cylindrical tube is achieved. The microwave streamer discharge is able to ignite air/propane mixtures at a low initial temperature and atmospheric pressure. Ignition of the air/propane mixture by a subcritical streamer discharge yields more than fourfold, from $5 \mathrm{~m} / \mathrm{s}$ to $150 \mathrm{~m} / \mathrm{s}$, increase in combustion speed compared to conventional spark ignition. A possibility of achievement of flame speeds larger than 100 $\mathrm{m} / \mathrm{s}$, corresponding to deflagration to detonation transition, is demonstrated. A major cause of the flame acceleration is an increase in the flame surface area and thereby the total heat release rate.

The more rapid combustion with streamer discharges could be exploited in practical combustion devices. Increase in flame surface, caused by the large ignition area, is one of the potential methods in improving combustion efficiency by reducing the burning time in the propulsion systems. These results suggest improved ignition devices for internal combustion engines, premixed gas turbines and pulse detonation engines.

\section{ACKNOWLEDGMENT}

This work was financially supported by the Ministry of Education and Science of Russian Federation (agreement No 14.577.21.0277, unique identifier of applied scientific research RFMEFI57717X0277).

\section{REFERENCES}

[1] Y. Ju, K. Maruta, "Microscale combustion: technology development and fundamental research", Progress in Energy and Combustion Science, vol. 37, no. 6, pp. 669-715, 2011.

[2] C. D. Cathey, T. Tang, T. Shiraishi, T. Urushihara, A. Kuthi, M. A. Gundersen, "Nanosecond plasma ignition for improved performance of an internal combustion engine", IEEE Transactions on Plasma Science, vol. 35 , no. 6, pp. 1664-1668, 2007

[3] I. Matveev, S. Matveeva, S. Serbin, "Design and preliminary result of the plasma assisted tornado combustor", AIAA Paper, 2007-5628, 2007.

[4] A. M. Davydov, S. I. Gritsinin, I. A. Kossyi, Y. M. Shikhman, V. A. Vinogradov, "Application of MW plasma generator for ignition of kerosene/air mixture", IEEE Transactions on Plasma Science, vol. 36, no. 6, pp. 2909-2917, 2008.

[5] H. Do, M. G. Mungal, M. A. Cappelli, "Jet flame ignition in a supersonic crossflow using a pulsed nonequilibrium plasma discharge", IEEE Transactions on Plasma Science, vol. 36, no. 6, pp. 2918-2923, 2008.

[6] I. Matveev, S. Serbin, "Investigation of a reverse-vortex plasma assisted combustion system", Proceedings of the ASME Heat Transfer Summer Conference, Puerto Rico, USA, HT2012-58037, pp. 133-140, 2012.
[7] S. Dresvin, S. Zverev, D. Ivanov, I. Matveev, "High frequency induction plasma torches", in Plasma Assisted Combustion, Gasification, and Pollution Control. Volume I. Methods of Plasma Generation for PAC. Outskirts Press, 2013, pp. 373-462.

[8] B. Wolk, A. DeFilippo, J.-Y. Chen, R. Dibble, A. Nishiyama, Y. Ikeda, "Enhancement of flame development by microwave-assisted spark ignition in constant volume combustion", Combustion and Flame, vol. 160, no. 7, pp. 1225-1234, 2013.

[9] T. X. Phuoc, "Single-point versus multi-point laser ignition: experimental measurements of combustion times and pressures", Combustion and Flame, vol. 122, no. 4, pp. 508-510, 2000.

[10] A. Hossain, N. Oshima, Y. Nakamura, M. Oshima, "Numerical investigation of the effect of ignition area on the subsequent flame propagation behavior", Journal of Thermal Science and Technology, vol. 4, no. 2, pp 214-225, 2009

[11] M. A. Nettleton, "Gaseous detonations: their nature, effects and control". Springer Science and Business Media, 2012.

[12] A. Starikovskiy, A. Rakitin, "Plasma-assisted ignition and deflagrationto-detonation transition", AIAA Paper, 2015-1601, 2015.

[13] A. P. Napartovich, I. V. Kochetov, S. B. Leonov, "Calculation of the dynamics of ignition of an airhydrogen mixture by nonequilibrium discharge in a high-velocity flow", High Temperature, vol. 43, no. 5, pp. 673-679, 2005

[14] A. M. Starik, V. E. Kozlov, N. S. Titova, On the influence of singlet oxygen molecules on the speed of flame propagation in methane-air mixture", Combustion and Flame, vol. 157, no. 2, pp. 313-327, 2010.

[15] N. A. Popov, "The effect of nonequilibrium excitation on the ignition of hydrogen-oxygen mixtures", High Temperature, vol. 45, no. 2, pp. 261-279, 2007.

[16] S. B. Leonov, D. A. Yarantsev, "Plasma-induced ignition and plasmaassisted combustion in high-speed flow", Plasma Sources Science and Technology, vol. 16, no. 1, pp. 132-139, 2007.

[17] G. Correale, A. Rakitin, A. Nikipelov, S. Pancheshnyi, I. Popov, A. Starikovskiy, T. Shiraishi, T. Urushihara, M. Boot, "Non-equilibrium plasma ignition for internal combustion engines", SAE Technical Paper, 2011-24-0090, 2011

[18] J. K. Lefkowitz, P. Guo, T. Ombrello, S.-H. Won, C. A. Stevens, J. L. Hoke, F. Schauer, Y. Ju, "Schlieren imaging and pulsed detonation engine testing of ignition by a nanosecond repetitively pulsed discharge", Combustion and Flame, vol. 162, no. 6, pp. 2496-2507, 2015.

[19] J. K. Lefkowitz, T. Ombrello, "Study of nanosecond pulsed high frequency discharge ignition in a flowing methane/air mixture", AIAA Paper, 2017-1777, 2017.

[20] M. Uddi, H. Guo, W. Sun, Y. Ju, "Studies of $\mathrm{C}_{2} \mathrm{H}_{6} /$ air and $\mathrm{C}_{3} \mathrm{H}_{8} /$ air plasma assisted combustion kinetics in a nanosecond discharge", AIAA Paper, 2011-970, 2011.

[21] E. Carbone, N. Sadeghi, E. Vos, S. Hűubner, E. van Veldhuizen, J. van Dijk, S. Nijdam, G. Kroesen, "Spatio-temporal dynamics of a pulsed microwave argon plasma: ignition and afterglow", Plasma Sources Science and Technology, vol. 24, no. 1, 015015, 2015.

[22] A. I. Shishpanov, A. V. Meshchanov, S. A. Kalinin, Y. Z. Ionikh, "Processes of discharge ignition in long tubes at low gas pressure", Plasma Sources Science and Technology, vol. 26, no. 6, 065017, 2017.

[23] I. Esakov, L. Grachev, K. Khodataev, D. van Wie, "The linear electromagnetic vibrator as the initiator of electric breakdown of air in deeply subcritical field of quasioptical microwave beam", AIAA Paper, 20111151, 2011.

[24] T. Shiraishi, T. Urushihara, M. Gundersen, "A trial of ignition innovation of gasoline engine by nanosecond pulsed low temperature plasma ignition", Journal of Physics D: Applied Physics, vol. 42, no. 13, 135208, 2009. 